\title{
MRT der Rotatorenmanschette - ein Update
}

\section{NMR of the rotator cuff - an update}

\author{
K.-F. Kreitner, A. Mähringer-Kunz
}

\section{Übersicht}

\section{Einleitung}

Anatomische Vorbemerkungen

MR-Bildgebung

Veränderungen der Rotatorenmanschette

Postoperative Bildgebung

Als Abonnent der Radiologie up2date

und Nutzer von Thieme-connect

finden Sie einen Podcast unter www.thieme-connect.de/products

\section{Zusammenfassung}

Die Rotatorenmanschette setzt sich aus den Sehnen der Mm. subscapularis, supraspinatus, infraspinatus und teres minor zusammen. Aufgrund

\section{Einleitung}

Die Rotatoren des Schultergelenks zählen zu den wichtigsten aktiven Stabilisatoren, indem sie den Humeruskopf bei den Bewegungen der Schulter und des Schultergürtels in der Gelenkpfanne zentrieren. Zusätzlich ermöglichen sie Rotationsbewegungen im Schultergelenk und - als Gegenspieler u. a. der Mm. deltoideus und teres major - Ab- und Adduktionsbewegungen der Schulter. Damit unterliegen die Sehnen der Rotatoren während des gesamten Lebens mehr oder weniger starken Beanspruchungen [1,2]. Dies erklärt, warum Erkrankungen der Rotatorenmanschette mit die häufigste Ursache von Schmerzen und Dysfunktionen des Schultergelenks im Erwachsenenalter sind. Ungeachtet der Fortschritte bei der sonografischen Beurteilung spielt die MR-Bildgebung nach wie vor eine wichtige Rolle, weil das Verfahren nicht nur detailliert die Rotatorenmanschette selbst darstellt, sondern es auch ihrer vielfältigen Funktionen unterliegen die Sehnen der Rotatoren während des gesamten Lebens mehr oder weniger starken Beanspruchungen. Dies erklärt, warum Erkrankungen der Rotatorenmanschette mit die häufigste Ursache von Schmerzen und Dysfunktionen des Schultergelenks im Erwachsenenalter sind. Ungeachtet der Fortschritte bei der sonografischen Beurteilung ist die MRT nach wie vor das wichtigste bildgebende Verfahren in der Beurteilung von Veränderungen der Rotatorenmanschette. Sie ermöglicht eine differenzierte Diagnostik, die letzten Endes Grundlage einer adäquaten Behandlung ist. Im vorliegenden Beitrag sollen aktuelle Konzepte hinsichtlich Anatomie und MR-Bildgebung vorgestellt werden. Nach Besprechung der Anatomie wird auf die normale und pathologisch veränderte Rotatorenmanschette und abschließend auf die postoperative Bildgebung eingegangen. ermöglicht, das Schultergelenk einschließlich der ossären und intraartikulären Strukturen umfassend zu beurteilen. Die vorliegende Arbeit soll einen Überblick über aktuelle Konzepte bei der MR-Bildgebung der Rotatorenmanschette verschaffen.

\section{Anatomische Vorbemerkungen}

\section{Sehnen der Rotatorenmanschette}

Die Rotatorenmanschette setzt sich von anterior nach posterior zusammen aus den Sehnen der Mm. subscapularis, supraspinatus, infraspinatus und teres minor (Abb. 1). Kennzeichen dieser Muskeln ist der Ursprung im Bereich der Skapula und der Ansatz am Humeruskopf $[2,3,4]$. 
Abb. 1 Rotatorenmanschettensehnen. Die anguliertsagittale T1w MRArthrografie zeigt die normale Anatomie der Rotatorenmanschette: $B$ und weißer Pfeil = Bizepssehne, Isp= Infraspinatussehne, Ssc $=$ Subskapularis sehne, Ssp = Supraspinatussehne, Tm=Teres-minorSehne, Doppelpfeil $=$ Rotatorenintervall.

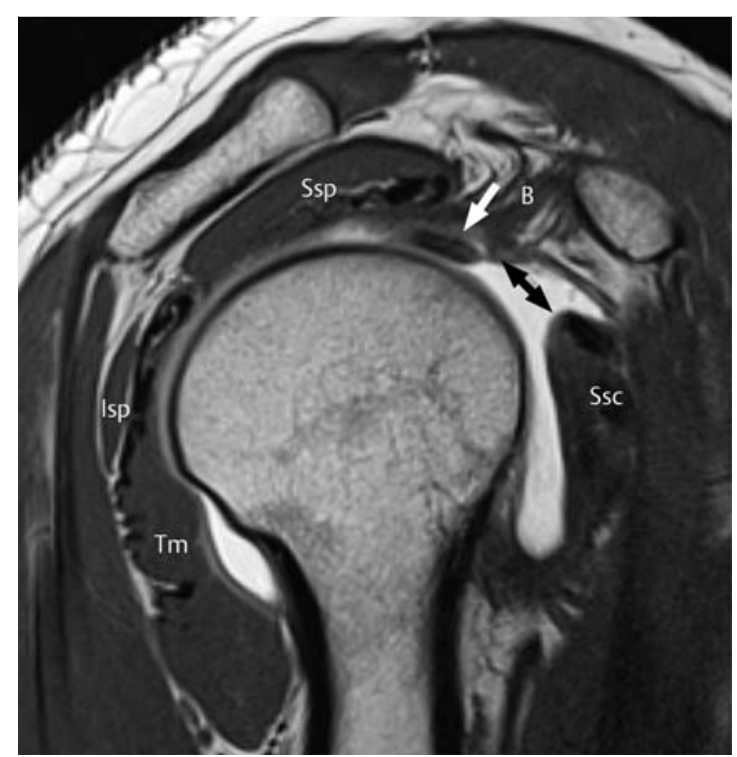

M. subscapularis. Der M. subscapularis entspringt von den medialen Dreivierteln der Fossa subscapularis, seine Muskelfasern ziehen nach lateral und die oberen zwei Drittel gehen auf Höhe des Glenoids in die Sehne über, die dann am Tuberculum minus ansetzt. Das kaudale Drittel des M. subscapularis hat einen direkten „muskulären“ Ansatz am Collum chirurgicum. Der Ansatzbereich am Tuberculum minus ist trapezförmig mit der breiteren Seite kranial und verjüngt sich nach kaudal. Neuere Untersuchungen haben gezeigt, dass die am weitesten kranial gelegenen Sehnenanteile über den Sulcus intertubercularis ziehen und am Tuberculum majus inserieren. Dort verschmelzen sie mit Fasern der Supraspinatussehne. Auf diese Weise trägt dieser Sehnenanteil zum Aufbau des Rotatorenintervalls bei (s.u.) $[3,4]$.

M. supraspinatus. Der M. supraspinatus entspringt von der Fossa supraspinata und der Oberfläche der Spina scapulae und setzt an der oberen Facette des Tuberculum majus an. Die Supraspinatussehne besteht aus 2 Anteilen: Der vordere Anteil ist lang und dick, der dorsale kurz und dünn. Der Ansatzbereich der Sehne ist dreieckförmig, von variabler Größe und beansprucht lediglich die anteromediale Hälfte der Facette. Gleichzeitig ziehen ventrale Fasern der Sehne über den Sulcus intertubercularis, um am Tuberculum minus zu inserieren.

M. infraspinatus. Der M. infraspinatus entspringt mit seinem größeren Anteil von der Fossa infraspinata und dem kleineren Teil von der Unterfläche der Spina scapulae. Er inseriert an der mittleren und der posterolateralen Hälfte der oberen Facette des Tubuerculum majus. Sein Ansatzbereich weist - analog zum M. supraspinatus - ebenfalls eine variable Größe auf.

M. teres minor. Der M. teres minor entspringt unterhalb des M. infraspinatus von der Margo lateralis scapulae und inseriert an der unteren Facette des Tuberculum majus, einzelne Faserzüge der kaudalen Anteile setzen direkt am Collum chirurgicum an $[2,5]$.

\section{Rotatorenintervall}

Mit dem Rotatorenintervall wird üblicherweise die Lücke zwischen dem Oberrand der Subskapularissehne und dem Vorderrand der Supraspinatussehne bezeichnet $[3,6]$. Es handelt sich um einen dreieckförmig geformten Raum, der medial von der Basis des Korakoidfortsatzes begrenzt wird und nach lateral in den Sulcus intertubercularis und das Lig. transversum humeri ausläuft. Leitstruktur des Rotatorenintervalls ist der intraartikuläre Anteil der langen Bizepssehne, die von einem Ausläufer der ventralen Gelenkkapsel umscheidet wird. Dieser Ausläufer wird von den Ligg. coracohumerale und glenohumerale superius verstärkt, die den Aufhängeapparat der langen Bizepssehne („Pulley“) bilden. Läsionen des Pulleys der langen Bizepssehne wie auch des Rotatorenintervalls gehen oft mit Läsionen der Subskapularis- und (weniger häufig) der Supraspinatussehne einher.

Die Rotatorenmanschette der Schulter wird von anterior nach posterior gebildet aus den Sehnen der Mm. subscapularis, supraspinatus, infraspinatus und teres minor. Die Lücke zwischen dem Oberrand der Subskapularis- und dem Vorderrand der Supraspinatussehne wird als Rotatorenintervall bezeichnet. Anatomische Leitstruktur ist die lange Bizepssehne mit ihrem Halteapparat („Pulley“).

\section{Aufbau der Sehnen}

Die Sehnen weisen einen differenzierten Aufbau aus Faszikeln auf, die aus Kollagenfibrillen bestehen und von Bindegewebe umgeben sind. Synoviale Zellen produzieren Proteoglykan 4, das auch als Lubrizin bezeichnet wird und ein Gleiten der Faszikel gegeneinander ermöglicht [7]. Die Abnahme des Lubrizins mit zunehmendem Alter führt zu einer erhöhten Friktion der Faszikel gegeneinander und ermöglicht Delaminationen der Sehnen, die sich zunächst in Form von intramuskulären Zystenbildungen in der MRT zu erkennen geben können (Abb. 2) [8]. 


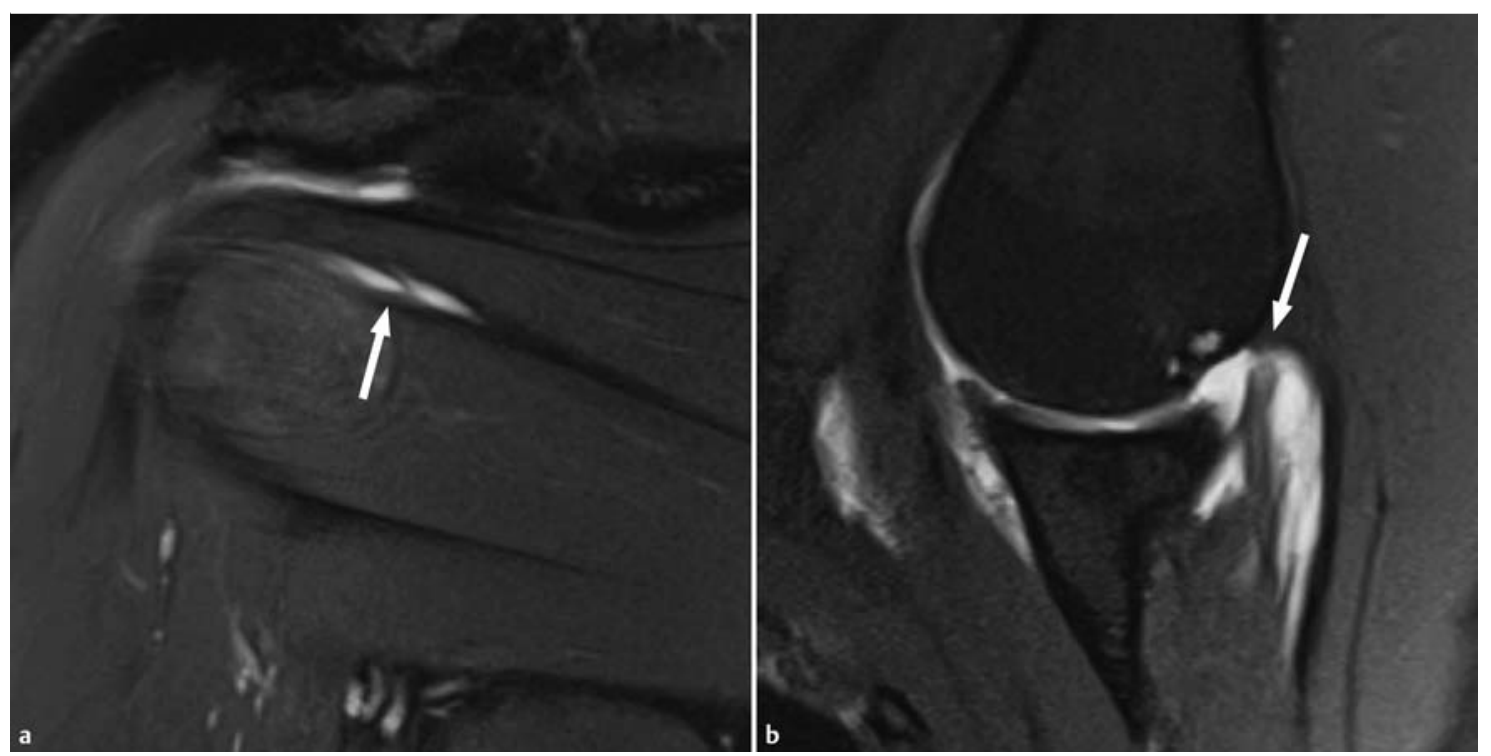

Abb. 2 Patientin mit einer Zyste am Oberrand des M. infraspinatus. a Die protonendichtegewichtete anguliert-koronare Aufnahme zeigt eine zystische Läsion am Oberrand der Infraspinatussehne (Pfeil). b In der Abduktions- und Außenrotationsaufnahme (ABER) demarkiert sich eine kleine partielle, posterosuperior gelegene, delaminierte Ruptur der Supraspinatussehne als Ursache der Zyste (Pfeil).
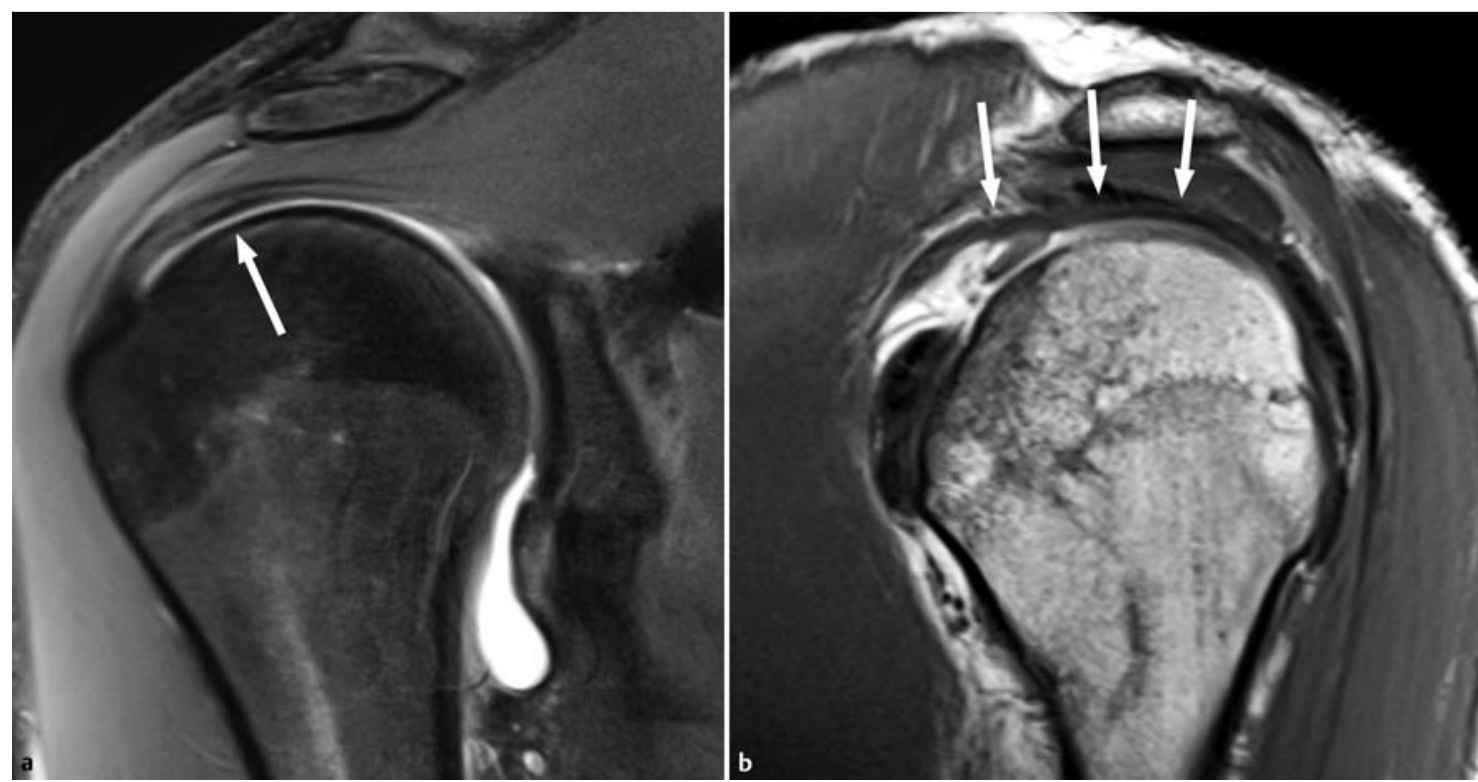

Abb.3 Abgrenzbarkeit des „rotator cable“ (Pfeile). T1w Aufnahmen mit und ohne Fettsuppression einer direkten MR-Arthrografie. a Anguliert-koronare Aufnahme. b Anguliert-sagittale Aufnahme.

Im Bereich der Supra- und Infraspinatussehne können darüber hinaus 5 verschiedene histologische Schichten von außen nach innen unterschieden werden [2]:

- Bei der äußersten Schicht handelt es sich um den oberflächlichen Ausläufer des Lig. coracohumerale.

- Daran schließen sich 2 Schichten mit mehr oder weniger dicht gepackten Sehnenfasern an, die zum jeweiligen Ansatz am Humeruskopf ziehen.

- Die nachfolgende 4. Schicht besteht zum einen aus lockerem Bindegewebe, zum anderen aus dicken Bändern von Kollagenfasern, die senkrecht zum Ver- lauf der Supra- und Infraspinatussehnen ausgerichtet sind und dem tiefen Ausläufer des Lig. coracohumerale entsprechen. Diese bandförmige Struktur ist seit den 1990er-Jahren bekannt und wird im Allgemeinen als „rotator cable“ bezeichnet $[8,9]$. Es findet sich auf koronaren Schnittbildern lateral der „kritischen“, weil minderdurchbluteten Zone der betreffenden Sehnen, die auch als „crescent zone“ bezeichnet wird (Abb.3). Funktionell wurde diese Bandverdickung und ihre Verbindungen zur Supra- und Infraspinatussehne mit einer Hängebrückenkonstruktion ver- 
glichen, bei der das „rotator cable“ zu einer merklichen Reduktion von Scher- und Zugkräften der Sehnen führt [10]. Experimentelle Untersuchungen haben gezeigt, dass sich Rupturen der Rotatorenmanschette bei intaktem Kabel biomechanisch weniger auswirken als solche mit Ruptur des Kabels [11]. Die Dicke des Kabels ist durchaus Schwankungen unterworfen und liegt meist bei etwa $1 \mathrm{~cm}$. Es kann am besten auf koronaren MR-Bildern abgegrenzt werden, die Häufigkeitsangaben schwanken zwischen 60 und 75\% bei intakter Rotatorenmanschette und erreichen $92 \%$ bei Patienten mit Rotatorenmanschettenrupturen und indirekter MR-Arthrografie [8, $9,12]$.

- Bei der innersten Schicht handelt es sich um die Kapsel des Schultergelenks.

Die Sehnen der Rotatoren weisen einen hochorganisierten Aufbau aus Bindegewebsfaszikeln auf, deren gegenseitige Verschieblichkeit durch Lubrizin gewährleistet wird. Das zumeist abgrenzbare „rotator cable“ macht aus der Rotatorenmanschette im Bereich der Supra- und Infraspinatussehne eine Hängebrückenkonstruktion und hilft, Zug- und Scherbelastungen zu minimieren.

\section{MR-Bildgebung}

\section{Arthrografieverfahren}

Grundsätzlich kommen bei der MR-Untersuchung der Rotatorenmanschette neben der nativen MRT 2 MR-Arthrografieverfahren in Betracht:

- die direkte MR-Arthrografie mit Injektion eines etwa 2 mmolar verdünnten, gadoliniumhaltigen Kontrastmittels in das Gelenk

- die indirekte MR-Arthrografie mit intravenöser Injektion eines gadoliniumhaltigen Kontrastmittels

Direkte MR-Arthrografie. Die direkte MR-Arthrografie ist der nativen MRT bei der Differenzierung zwischen partieller und kleiner vollständiger Rotatorenmanschettenruptur überlegen [13]. Durch die direkte MR-Arthrografie erhöht sich die Sensitivität im Nachweis partieller Rupturen von 64\% auf etwa $85 \%$.

Indirekte MR-Arthrografie. Inzwischen liegen vermehrt auch Mitteilungen über die Treffsicherheit der indirekten MR-Arthrografie vor, die mit der der direkten MRArthrografie vergleichbar sind [12,14]. Bei der indirekten MR-Arthrografie bewegt der Patient seine Schulter nach der intravenösen Kontrastmittelinjektion etwa
10 - 15 Minuten lang, bevor die MRT durchgeführt wird. Der arthrografische Effekt bei der indirekten MR-Arthrografie profitiert ganz eindeutig von einem vorhandenen Gelenkerguss und kann deshalb bei einem fehlenden Erguss nicht oder nur sehr gering ausgeprägt sein.

\section{Untersuchung}

Ebenen. Auch bei der Klärung einer Veränderung der Rotatorenmanschette sollte das Schultergelenk in allen 3 Ebenen untersucht werden:

- Die axiale Ebene umfasst dabei kranial das gesamte AC-Gelenk und kaudal das gesamte Glenoid.

- Die anguliert-koronare Schnittebene verläuft parallel zur Supraspinatussehne und senkrecht zur Gelenkfläche des Glenoids.

- Die anguliert-sagittale Ebene verläuft senkrecht zur anguliert-koronaren Ebene und sollte lateral den Ansatz der Sehnen am Humeruskopf und medial die Muskelbäuche der Rotatoren umfassen.

Protokolle. Zumeist werden schnelle Spin-EchoSequenzen verwendet. Zur Beurteilung einer Veränderung der Rotatorenmanschette sind Intermediär- und T2w Sequenzen unabdingbar, wobei die Intermediärsequenzen eine Echozeit $>37 \mathrm{~ms}$ aufweisen und mit einer Fettsuppression kombiniert sein sollten. Die MRProtokolle hängen neben der Fragestellung des Zuweisers auch davon ab, welche Soft- und Hardware des MR-Scanners vorhanden ist. Unsere aktuellen Standardprotokolle bei 3 $\mathrm{T}$ sind in Tab. 1 zusammengefasst.

ABER-Position. Fakultativ können auch Aufnahmen in Abduktions- und Außenrotationsstellung des Arms (sog. ABER-Position) angefertigt werden. Durch diese Untersuchungstechnik wird neben dem vorderen unteren Pfannenrand mit dem ansetzenden glenohumeralen Ligamenten die posterosuperiore Rotatorenmanschette besser beurteilbar [15], die insbesondere bei Mikroinstabilitäten pathologische Veränderungen aufweisen kann. Nachteilig bei der Akquisition von Sequenzen in ABER-Position ist neben dem Umlagern des Patienten die Tatsache, dass die dedizierte Schulterspule nicht zur Signaldetektion verwendet werden kann. Aktuell werden Aufnahmen in ABER-Position nicht routinemäßig zur Klärung einer veränderten Rotatorenmanschette akquiriert (Abb.2b). 
Tabelle 1

MRT-Untersuchungsprotokolle zur Klärung von Veränderungen der Rotatorenmanschette.

\begin{tabular}{|c|c|c|c|c|c|c|}
\hline Sequenz & Orientierung & Fettunterdrückung & TR (ms) & TE (ms) & Schichtdicke (mm) & FOV $(\mathrm{mm})$ \\
\hline \multicolumn{7}{|l|}{ Native MRT bei $3 \mathrm{~T}$} \\
\hline PD TSE/FSE & anguliert koronar & ja & 4400 & 38 & 3 & 160 \\
\hline PD TSE/FSE & transversal & ja & 3000 & 41 & 3 & 160 \\
\hline T1 TSE/FSE & anguliert sagittal & nein & 600 & 11 & 3 & 140 \\
\hline T2 TSE/FSE & anguliert sagittal & ja & 4000 & 72 & 3 & 160 \\
\hline \multicolumn{7}{|c|}{ Direkte MR-Arthrografie bei $3 \mathrm{~T}$} \\
\hline PD TSE/FSE & anguliert koronar & ja & 4400 & 42 & 3 & 160 \\
\hline T1 TSE/FSE & anguliert koronar & ja & 660 & 10 & 3 & 160 \\
\hline T1 TSE/FSE & transversal & ja & 720 & 10 & 3 & 160 \\
\hline T1 TSE/FSE & anguliert sagittal & nein & 600 & 11 & 3 & 140 \\
\hline fakultativ: T1 TSE/FSE & ABER & ja & 660 & 10 & 3 & 160 \\
\hline
\end{tabular}

Die Rotatorenmanschette kann MR-tomografisch sowohl nativ als auch mittels direkter oder indirekter MR-Arthrografie beurteilt werden. Kontrastverstärkte Untersuchungstechniken sind der nativen Untersuchung insbesondere im Nachweis partieller Rupturen überlegen. Wichtigster Sequenztyp sind schnelle Turbo-Spin-Echo-Sequenzen, die in allen 3 Ebenen angefertigt werden sollten.

\section{Veränderungen der Rotatorenmanschette}

Bei den Veränderungen der Rotatorenmanschette wird generell zwischen Tendinopathie, Partialruptur und kompletter, d.h. transtendinöser Ruptur unterschieden $[2,3,16]$.

\section{Tendinopathien}

Charakteristika. Die Tendinopathie ist gekennzeichnet durch eine Unterbrechung von Kollagenfasern mit nachfolgender Abnahme des Typ-I-Kollagens, mukoide Degenerationen, eine vermehrte Akkumulation von
Glykosaminoglykanen sowie u.U. einen vermehrten Wassergehalt.

MR-Bildgebung. In der MR-Bildgebung sind Tendinopathien charakterisiert durch eine Signalintensitätserhöhung auf Sequenzen mit kurzen Echozeiten (T1-, prototonendichtegewichtet), aber fehlender Anhebung des MR-Signals auf T2w Sequenzen. Wichtigster „pitfall“ in der Diagnostik einer Tendinopathie ist der "Magic-Angle“-Artefakt: Er entsteht dadurch, dass geordnete anatomische Strukturen wie Sehnen und Bänder (Kollagenfasern) ein erhöhtes Signal aufgrund minimaler Dipolinteraktionen aufweisen, wenn ihre Ausrichtung einen Winkel von $55^{\circ}$ gegenüber dem statischen Magnetfeld einnimmt. Den „Magic-Angle“Artefakt findet man am häufigsten im Bereich der Supraspinatussehne kurz vor dem Ansatz am Tuberculum majus. Um diesem Artefakt nicht aufzusitzen, sollten Sequenzen (intermediär gewichtete) in anguliertkoronarer Schnittführung eine Echozeit $\geq 37 \mathrm{~ms}$ aufweisen (Abb.4). Mit zunehmendem Wassergehalt wie z. B. bei entzündlichen Reizungen steigt auch die Signalintensität der Sehnen auf T2w Aufnahmen. Gleichzeitig kann eine unterschiedliche oder zunehmende Dicke der Sehne auf eine pathologische Veränderung der Sehne hinweisen (Abb.5). 
Abb. 4 Einfluss der Echozeit auf die Signalanhebung der Supraspinatussehne auf anguliert-koronaren Aufnahmen. a Bei einer Echozeit von $20 \mathrm{~ms}$ findet sich ein typisches „Magic-Angle“-Phänomen (Pfeil), das eine Signalanhebung der Sehne vortäuscht. b Bei einer Echozeit von $40 \mathrm{~ms}$ normale hypointense Darstellung der Sehne (Pfeil) im gesamten Verlauf.
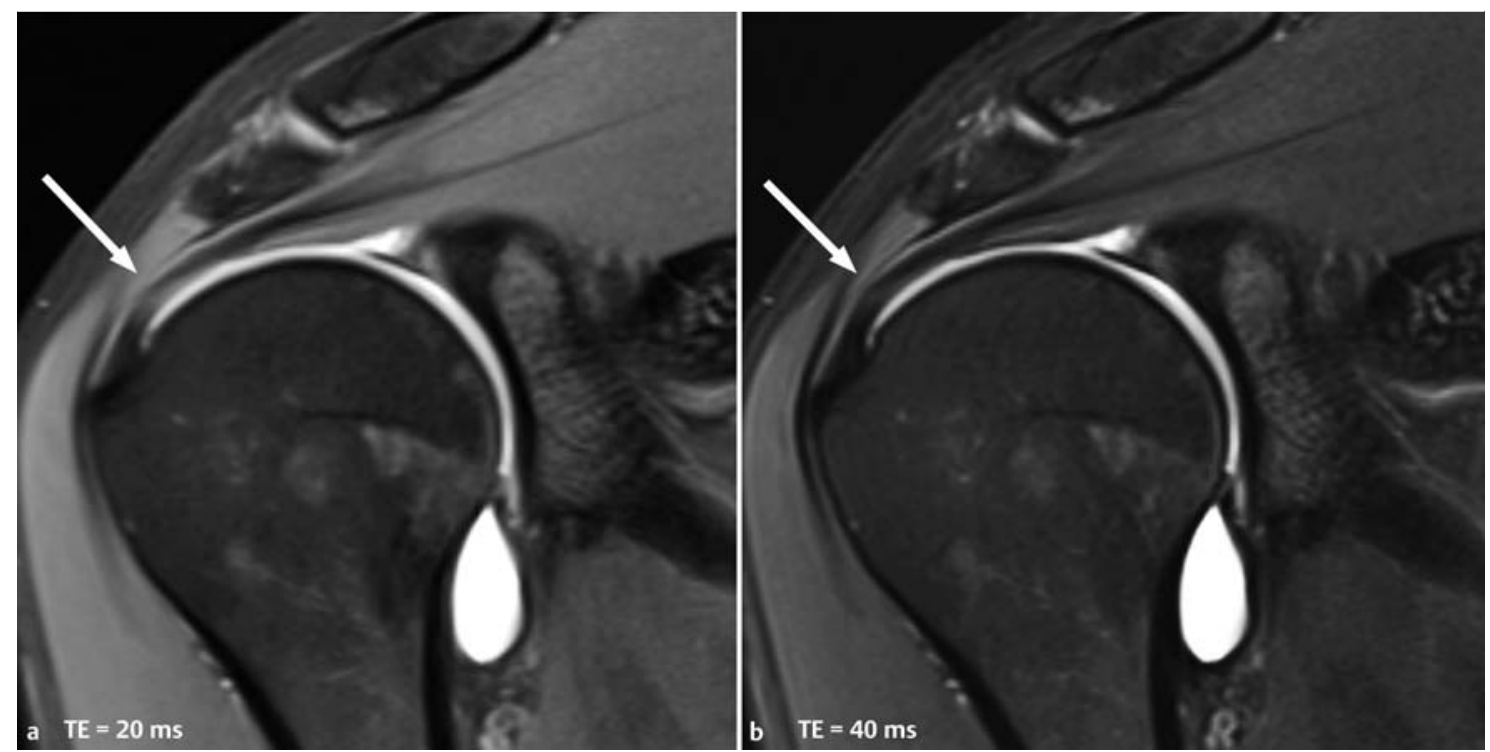

Abb. 5 Tendinitis der Supraspinatussehne mit Auftreibung und deutlicher Signalanhebung der Sehne, aber ohne Unterbrechung der Sehnenkontur (Pfeil). Begleitender Gelenkerguss sowie Erguss in der Bursa subacromialis-subdeltoidea. Intermediär-gewichtete TSE-Aufnahme.

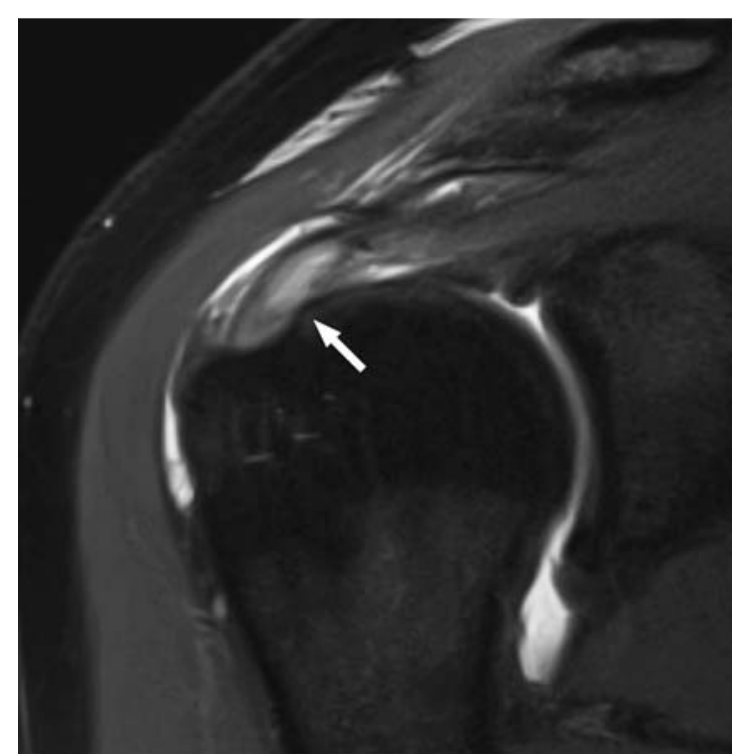

Tendinopathien und Tendinitiden führen zu einer erhöhten Signalintensität der betroffenen Sehnen. Dies muss aber gegenüber einem „Magic-Angle“Artefakt abgegrenzt werden, der sich durch Wahl von Sequenzen mit einer Echozeit $\geq 37$ ms verhindern lässt. Bei einer Tendinitis können nahezu flüssigkeitsäquivalente Signalanhebungen vorgefunden werden, ohne dass eine Unterbrechung der Sehnenkontinuität vorliegt.

\section{Partielle Rotatorenmanschettenrupturen}

\section{Typen}

Partielle Rotatorenmanschettenrupturen sind dadurch gekennzeichnet, dass die Ruptur nicht den gesamten Querschnitt der Sehne betrifft. Man unterscheidet gelenk- von bursaseitig gelegenen Partialrupturen sowie Partialrupturen, die innerhalb der Sehne auftreten (interstitielle Partialruptur) und weder Kontakt zur gelenk- noch zur bursaseitigen Oberfläche haben $[1,2,16]$. Gelenkseitige Teilrupturen sind etwa zweimal häufiger als bursaseitige; zumeist ist die Sehne des M. supraspinatus im ventralen Anteil betroffen. Intratendinöse Teilrupturen werden nach Sektionsbefunden sogar noch häufiger als gelenkseitige Partialrupturen gefunden, diese Beobachtungen sind aber bislang weder bildgebend noch operativ so bestätigt worden.

\section{- Lokalisation}

Patienten $>\mathbf{4 0}$ jahre. Partialrupturen werden in allen Altersgruppen beobachtet. Bei Patienten über 40 Jahren liegt die Rupturstelle aufgrund von Degenerationen zumeist in der „kritischen“, weil minderdurchbluteten Zone der Infra- und Supraspinatussehne ca. $1 \mathrm{~cm}$ medial des Ansatzbereichs. Gleichzeitig werden mehr bursaseitige Teilrupturen und auch Delaminationen beobachtet, letztere können mit intramuskulär gelegenen Zysten vergesellschaftet sein.

Jüngere Patienten. In den letzten Jahren wurde zunehmend Partialrupturen Beachtung geschenkt, die den Ansatz der Sehne mit einbeziehen und als „Footprint“Läsionen bezeichnet werden. Diese werden gehäuft bei jüngeren Patienten vorgefunden und sind häufig Folge 

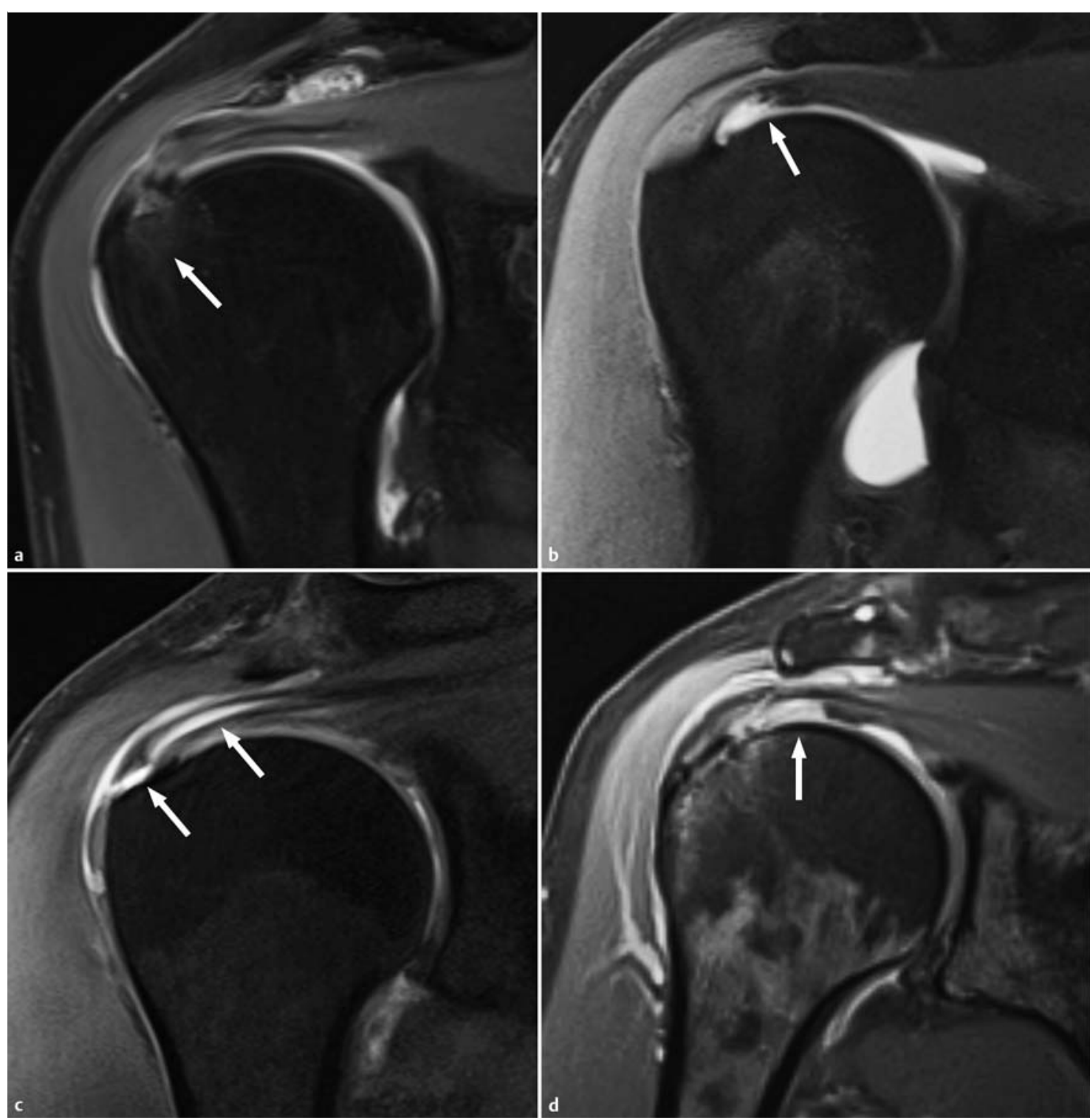

Abb. 6 Partialrupturen der Rotatorenmanschette (die definitionsgemäß nicht den gesamten Sehnenquerschnitt betreffen) auf T1- (b) und fettsaturierten intermediär-gewichteten Aufnahmen (a, c, d). a Interstitielle Partialruptur, CID-Läsion (CID = „concealed interstitial delamination“). Am Humeruskopf sind ödematöse Veränderungen nachweisbar (Pfeil). b PASTA-Läsion (PASTA= „partial articular-sided supraspinatus tendon avulsion“) in einer T1w fettunterdrückten MR-Arthrografieaufnahme: Der Pfeil zeigt auf die retrahierten gelenkseitig gelegenen Sehnenanteile, die Rupturstelle ist am Footprint der Sehne. $\mathbf{c}$ Bursaseitig gelegene „Reverse“ PAINT-Läsion (PAINT = „partial articular side lesion with interstitial extension“) in einer fettsupprimierten anguliert-koronaren Aufnahme. Die Pfeile zeigen den Eintritt der Flüssigkeit der Bursa am Footprint der Sehne mit Ausdehnung der Flüssigkeit nach medial und Delamination der Sehne.

d STAS-Läsion (STAS = „supraspinatus tendon articular side“) einer gelenkseitig gelegenen Partialruptur, die den Footprint der Sehne nicht mit einbezieht (Pfeil).

von Überkopfsportarten oder Traumen [16,17]. Diese Partialrupturen wurden aufgrund ihres Pathomechanismus weiter subklassifiziert und mit Akronymen belegt, die sich an der englischsprachigen Literatur orientieren und oft auch von Orthopäden benutzt werden [18]:

- Die CID-Läsion (CID = „, concealed interstitial delamination“) ist eine gedeckte intratendinöse Partialruptur am Sehnenansatz, bei der zusätzlich in etwa einem Viertel der Fälle ein Knochenmarködem im Tuberculum majus vorliegt (Abb.6a).

- Die PASTA-Läsion (PASTA = „partial articular-sided supraspinatus tendon avulsion“) ist eine gelenkseitige Partialruptur mit Beteiligung des tendoossären Übergangs (Abb.6b). Bei der „Reverse“ PASTA-Läsion liegt eine bursaseitige Partialruptur der Sehne ausgehend vom tendoossären Übergang vor; sie ist damit das Pendant zur PASTA-Läsion auf der Bursaseite. 


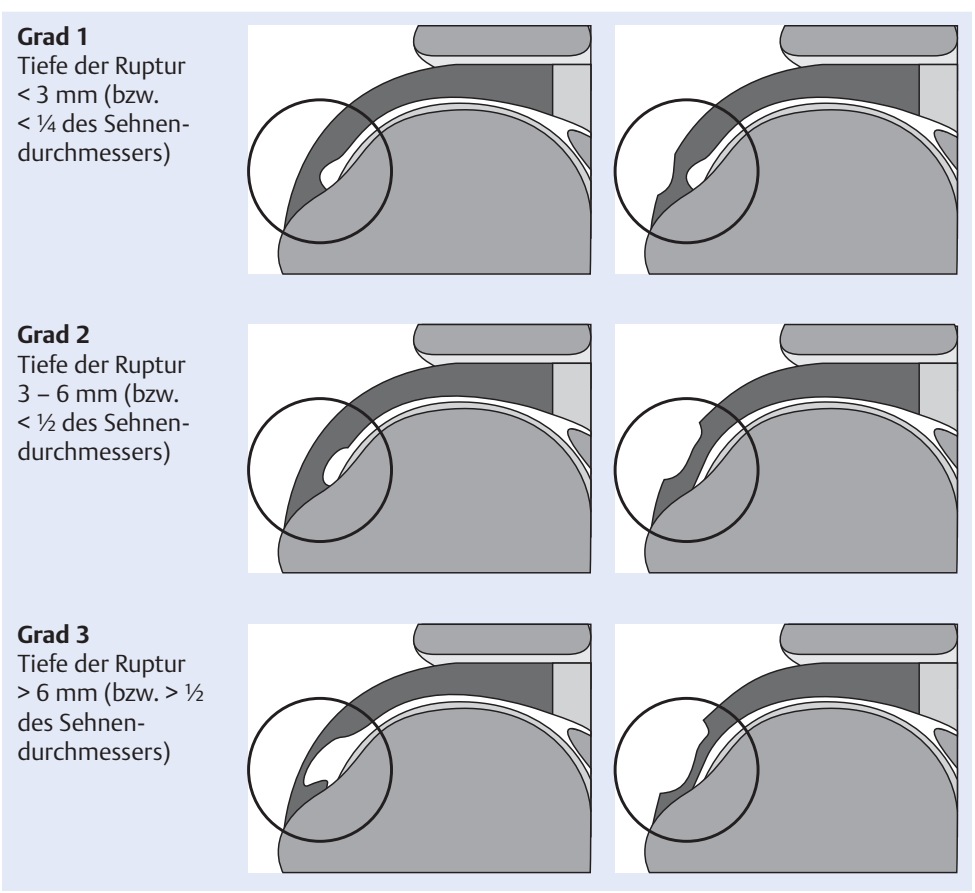

Abb. 7 Klassifikation von Partialrupturen hinsichtlich der Ausdehnung nach Ellman [nach $[18,19])$.

\section{Vorgehen}

Obwohl bislang noch wenig bekannt ist über den Spontanverlauf einer partiellen Rotatorenmanschettenruptur, gibt es die Empfehlung, dass drittgradige Partialrupturen operativ behandelt werden sollten. Bei den partiellen Rotatorenmanschettenrupturen unter Einbeziehung des Sehnenansatzes (Footprint-Läsionen) wird gerade auch bei jüngeren Patienten unabhängig von der Ausdehnung ein eher chirurgisches Vorgehen empfohlen.

Partielle Rotatorenmanschettenrupturen betreffen nicht den gesamten Querschnitt der Sehne. Man unterscheidet gelenk- von bursaseitig gelegenen sowie interstitielle Partialrupturen. Partialrupturen, die den Ansatz der Sehne mit einbeziehen, werden als „Footprint“-Läsionen bezeichnet. Diese treten bei jüngeren Patienten auf und werden eher einer operativen Therapie zugeführt.

\section{Komplette Rotatorenmanschettenrupturen}

\section{- Charakteristika}

Bei der kompletten oder transtendinösen Rotatorenmanschettenruptur ist der gesamte Sehnenquerschnitt von der Ruptur betroffen; in der englischsprachigen Literatur wird deshalb der Begriff „,full-thickness tear“ verwendet. Somit besteht beispielsweise bei einer Supra- oder Infraspinatussehnenruptur eine Verbindung zwischen dem Schultergelenk und der Bursa subacromialis-subdeltoidea.

Ursachen. Komplette Rotatorenmanschettenrupturen sind bei Patienten unter 55 Jahren oft Folge eines Traumas, bei Patienten über 55 Jahren eher Folge von Sehnendegenerationen.

Größe der Ruptur. Die Ruptur kann dabei stecknadelkopfgroß sein oder die gesamte Sehne betreffen, deshalb sollte die Größe der Ruptur im Befund angegeben werden; es wird empfohlen, die Größenausdehnung mediolateral und anteroposterior in Zentimetern anzugeben (Abb.8).

Retraktionsgrad. Zur Beschreibung des Retraktionsgrades wird insbesondere bei größeren Rupturen die Einteilung nach Patte angewendet [20]:

- Bei Grad 1 reicht der retrahierte Sehnenstumpf nicht bis zum Scheitelpunkt (Dom) des Humeruskopfes.

- Bei Grad 2 liegt der Sehnenstumpf zwischen dem Scheitelpunkt des Humeruskopfes und dem Glenoid.

- Bei Grad 3 ist der Sehnenstumpf bis zum Glenoid oder darüber hinaus retrahiert (Abb.9, Abb.10). 



Abb. 8 T1w, fettsaturierte anguliertkoronare (a) und transversale (b) MRArthrografie-Aufnahmen einer kompletten, delaminierten Ruptur der Supraspinatussehne (Pfeil), bei der die Ausdehnung sowohl mediolateral als auch anterior-posterior (Doppelpfeil) bestimmt werden kann. Retraktionsgrad 1 nach Patte.

Ebenso sollte das Vorhandensein von Delaminationen im Befund erwähnt werden.

\section{MR-Bildgebung}

Treffsicherheit. Die MRT besitzt eine hohe Treffsicherheit im Nachweis kompletter Rotatorenmanschettenrupturen: Mit der nativen MRT werden Sensitivitäten und Spezifitäten von 92\% bzw. 93\% erreicht, die direkte MR-Arthrografie weist eine Sensitivität von 95\% und Spezifität von 99\% auf. Neuere Arbeiten berichten über Sensitivitäten und Spezifitäten der indirekten MR-Arthrografie bei 3 T zwischen 92 und 100\% bzw. 94 und 100\% [12, 13,14]. Falsch negative Befunde bei der MRT sind zumeist Folge einer fibrösen Narbenplatte oder einer Verklebung an der Rupturstelle.

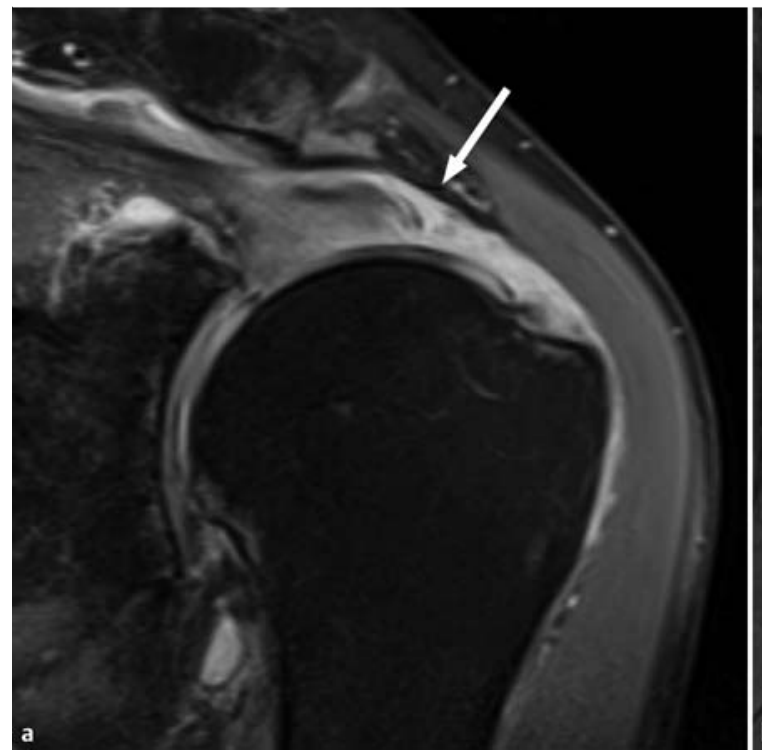

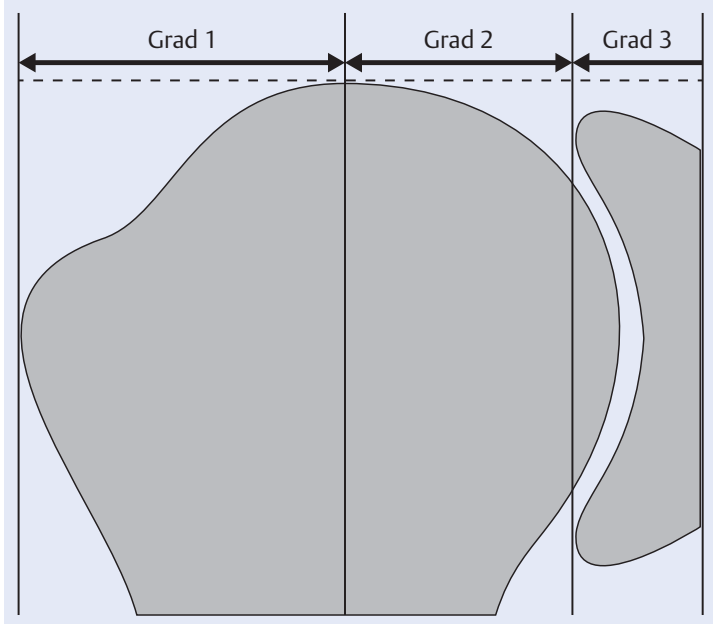



Abb. 9 Retraktionsgrad nach Patte bei kompletten Rotatorenmanschettenrupturen (nach [19]).

Abb. 10 Ausgedehnte Rotatorenmanschettenruptur mit vollständigem Sehnenabriss der Supraspinatussehne (a) und der Subskapularissehne (b) (Pfeil). Zusätzlich Abriss der langen Bizepssehne bei fehlender Darstellung im Sulcus intertubercularis (Stern). Protonendichtegewichtete, fettgesättigte Aufnahmen einer nativen MRT-Untersuchung. 
Tabelle 2

Klassifikation der fettigen Degeneration der Rotatorenmanschettenmuskeln nach Goutallier [21].

\begin{tabular}{|ll|}
\hline Grad & Befund \\
\hline Grad 0 & kein Fett, nur Muskulatur \\
\hline Grad I & vereinzelte Fettstreifen in der Muskulatur \\
\hline Grad II & deutliche fettige Degeneration, aber Fettmasse < Muskelmasse \\
\hline Grad III & fortgeschrittene fettige Degeneration, Fettmasse = Muskelmasse \\
\hline Grad IV & fortgeschrittene fettige Degeneration, Fettmasse $>$ Muskelmasse \\
\hline
\end{tabular}

Bei der kompletten Rotatorenmanschettenruptur ist der gesamte Sehnenquerschnitt von der Ruptur betroffen. Die Größe der Ruptur und das Ausmaß der Sehnenretraktion sollten im Befund festgehalten werden.

Atrophie und Verfettung. Rotatorenmanschettenrupturen können - je nach Ausmaß - zur Atrophie und Verfettung der betroffenen Muskeln führen. Klinisch und experimentell können diese schon nach 4 Wochen beobachtet werden. Atrophie und Verfettung der Rotatorenmanschettenmuskulatur spielen bei der Wahl des Operationsverfahrens eine große Rolle, weil rekon- struktive Verfahren nicht mehr infrage kommen, wenn die Muskulatur bereits in fortgeschrittenem Ausmaß atrophiert und verfettet ist:

- Die Klassifikation der fettigen Degeneration geht auf Goutallier zurück, der diese anhand von CT-Datensätzen etablierte (Tab.2) [21]. Diese Einteilung kann aber auch auf MRT-Untersuchungen übertragen werden. Aufgrund der besseren Gewebedifferenzierung mit der MRT ist das Verfahren genauer als die CT [22]; generell gilt, dass ab einem Degenerationsgrad 3 eine Sehnenrekonstruktion nicht mehr sinnvoll ist.

- Für die Erfassung einer Atrophie der Supraspinatussehnenmuskulatur wird das Tangentenzeichen verwendet [23]: Hierbei wird eine Verbindungslinie zwischen der kranialen Begrenzung von Spina scapulae und Processus coracoideus auf parasagittalen MRT-Aufnahmen gezogen und beurteilt, ob der Muskelbauch diese Tangente schneidet (negatives Tangentenzeichen) oder unterhalb der Tangente liegt (positives Tangentenzeichen). Für die Beurteilung des Tangentenzeichens sollte auf anguliert-sagittalen Aufnahmen das erste Bild gewählt werden, auf der die Spina scapulae in Kontakt mit der Skapula ist und so eine Y-Form bildet (Abb.11).


Abb.11 Normale und verfette sowie atrophierte Rotatorenmanschettenmuskulatur. a Die anguliert-sagittale T1w Aufnahme zeigt eine erhebliche Verfettung und Atrophie der Mm. supraspinatus und infraspinatus (Pfeile). Normale Darstellung des M. subscapularis (Ssc). b Positives Tangentenzeichen des M. supraspinatus bei einer kleinen kompletten Ruptur der Sehne (gleicher Patient wie in Abb. 8) mit diskreten Verfettungen der Sehne (Grad 1 nach Goutallier) wie auch im M. infraspinatus (Isp). Unauffälliger Befund des M. subscapularis. Anguliert-sagittale T1w Aufnahme. 

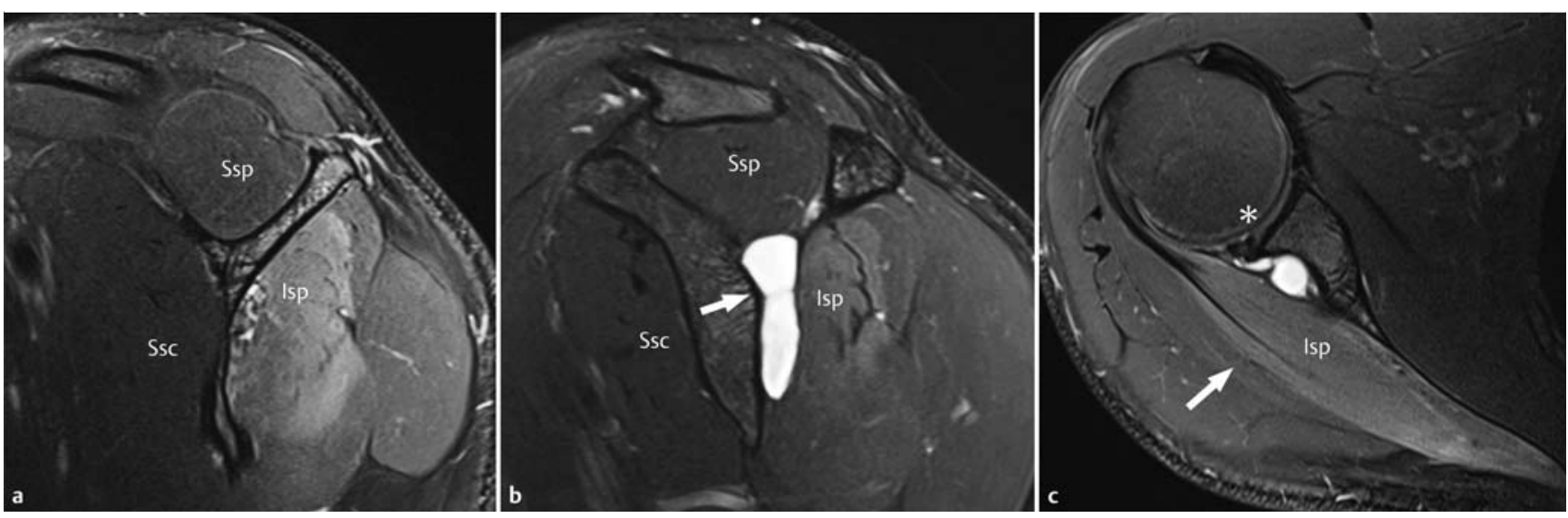

Abb. 12 Denervierungsödem des M. infraspinatus (Isp) (a-c) als Folge eines großen Ganglions (Pfeil) (b), das sich auf dem Boden eines Risses des posterosuperioren Labrums (Stern) (c) entwickelt hat und in der Incisura spinoglenoidalis auf den N. suprascapularis drückt (b, c). Intermediär- und protonendichtegewichtete, fettsupprimierte Aufnahmen einer nativen MRT-Untersuchung. Ssp= M. supraspinatus, Ssc = M. subscapularis, Isp=Infraspinatussehne.

Rotatorenmanschettenrupturen können zu Atrophie und Verfettung der betroffenen Muskeln führen. Zur Beurteilung der Verfettung hat sich die Einteilung nach Goutallier, zur Beurteilung der Atrophie das Tangentenzeichen nach Zanetti bewährt.

Zysten. In seltenen Fällen können innerhalb der Sehnen oder auch der dazugehörigen Muskeln Zysten beobachtet werden, deren Ausrichtung sich an der der Sehnen- bzw. Muskelfasern orientiert. Diese Zysten werden auf einen Defekt in der Oberfläche einer Rotatorensehne zurückgeführt, über den Gelenkflüssigkeit oder Flüssigkeit eines Schleimbeutels (z. B. Bursa subacromialis-subdeltoidea) austreten kann und konsekutiv Sehnen- oder Muskelfasern auseinanderdrängt [8]. In etwa der Hälfte der Fälle treten zystische Formationen bei einer Partialruptur der Rotatorenmanschette auf, deren Nachweis der nativen MRT entgehen kann (Abb.2). Solche intramuskulären Zysten sollten dabei nicht mit paralabralen Zysten oder Ganglien verwechselt werden, deren Grundlage ein Riss im Labrum glenoidale ist. Paralabrale Zysten können bei entsprechender Größe und Lage, klassischerweise ausgehend vom posterosuperioren Labrum zu einer Kompression nervaler Strukturen insbesondere im Bereich der Incisura spinoglenoidalis (N. suprascapularis) führen. Die Folge sind Denervierungen der Mm. infra- und supraspinatus, die sich zunächst in ödematösen Veränderungen der betroffenen Muskeln manifestieren (Abb.12). Klinisch sind diese Denervierungen nicht von primären Läsionen der Sehnen zu unterscheiden.

\section{Besonderheiten bei Läsionen des Musculus subscapularis}

Rupturen der Subskapularissehne sind deutlich seltener als die der Supraspinatussehne. Ihre Prävalenz betrug etwa $10 \%$ in einem arthroskopisch korrelierten Patientenkollektiv eines Zentrums für muskuloskelettale Bildgebung. Obwohl der M. subscapularis der Größte der Rotatoren des Schultergelenks ist, sind Läsionen seiner Sehne klinisch nur sehr schwierig zu diagnostizieren. Zusätzlich können sie auch bei der arthroskopischen Beurteilung leicht übersehen werden, wenn nicht gezielt nach ihnen gefahndet wird.

Einteilung. Die Läsionen der Sehne des M. subscapularis werden - anders als bei Läsionen der Supra- und Infraspinatussehne - in Oberrandläsionen, partielle und komplette Rupturen unterteilt [16,24,25]. Eine Oberrandläsion liegt vor, wenn das kraniale Drittel der Sehne von der Ruptur betroffen ist, bei einer Partialruptur ist die kraniale Hälfte und bei einer kompletten Ruptur die gesamte Sehneninsertion abgerissen (Abb. 10, Abb.13).

Ursachen. Subskapularissehnenrupturen können isoliert nach einem Trauma mit kräftiger Außenrotation des adduzierten Arms auftreten. Viel häufiger sind jedoch eine Beteiligung des kranialen Anteils der Subskapularissehne bei ausgedehnten Rupturen der Supraspinatussehne und Risse, die das Rotatorenintervall und seine begrenzenden Strukturen betreffen (Abb. 10, Abb. 14). In diesen Fällen sollte auch gezielt nach Läsionen (Tendinopathie, Medialverlagerung vor das Tuberculum minus, in das Glenohumeralgelenk) 
Abb. 13 Partialruptur der Subskapularissehne, T1w fettgesättigte Aufnahmen einer direkten MR-Arthrografie. a Gelenkseitig gelegene Retraktion einer Sehnenfasern (Pfeile). b In der anguliert-sagittalen Aufnahme Interposition von Kontrastmittel zwischen dem Tuberculum minus und der kranialen Hälfte der Sehne (Pfeil).
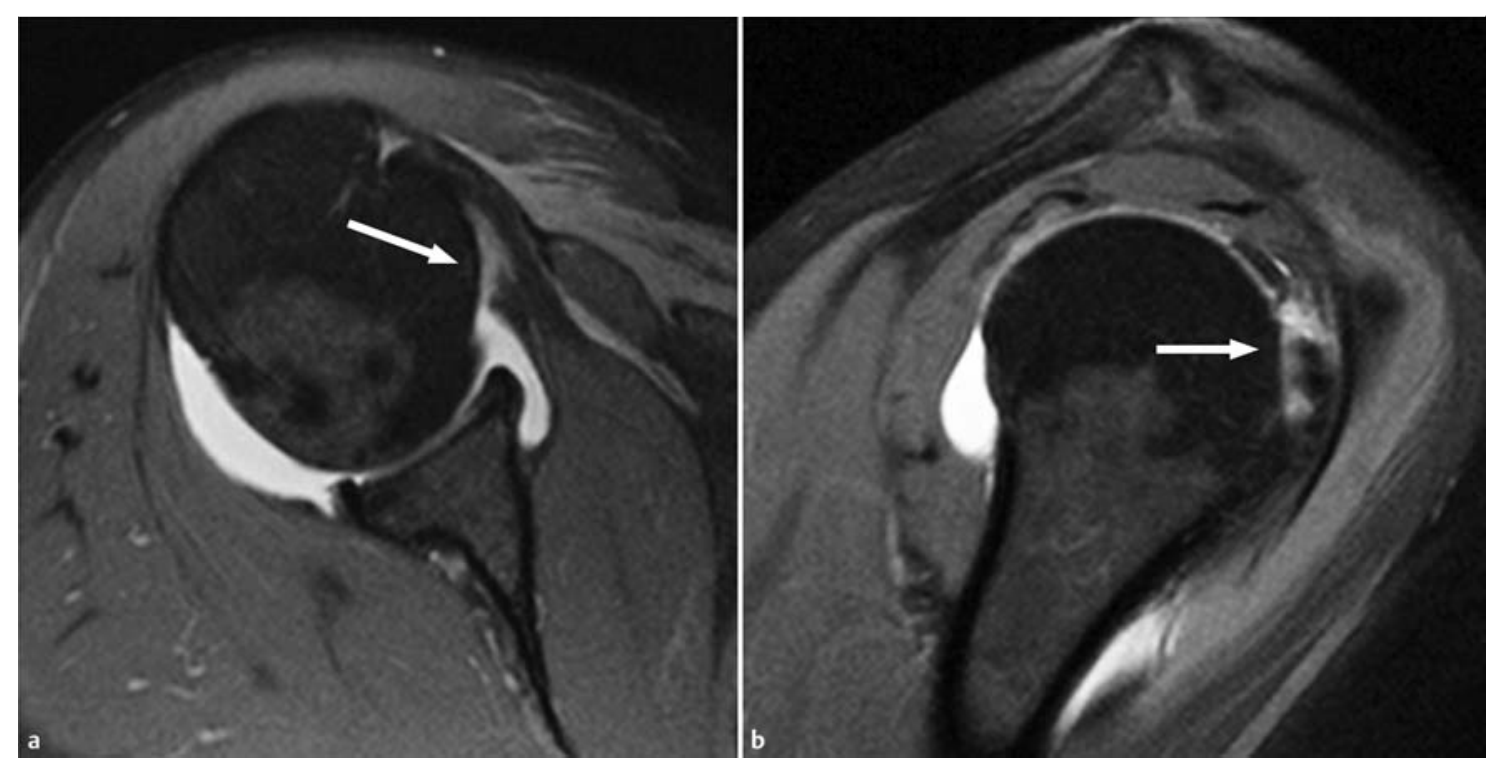

der langen Bizepssehne und seines Halteapparats („Pulley“) gefahndet werden.

MR-Bildgebung. Die Subskapularissehne sollte sowohl auf transversalen als auch auf den anguliert-sagittalen MRT-Aufnahmen sorgfältig analysiert werden; hier ist insbesondere auf Diskontinuitäten, umschriebene Signalanhebungen und Kaliberschwankungen zu achten. Bei der direkten MR-Arthrografie zeigt das Eindringen von Kontrastmittel in die Sehne eine Ruptur an. Die MR-Arthrografie erreicht eine Sensitivität von $91 \%$ und eine Spezifität von $86 \%$, die Sensitivität im Nachweis partieller Rupturen sinkt auf $63 \%$, wenn lediglich eine native MRT angefertigt wird. Der direkte Kontakt von Kontrastmittel und Tuberculum minus bei der direkten
MR-Arthrografie wie auch die fettige Atrophie des M. subscapularis besitzen eine hohe Spezifität (90$100 \%$ im Nachweis einer Subskapularisruptur, beide sind jedoch nicht ausreichend sensitiv.

\footnotetext{
Die Läsionen der Sehne des M. subscapularis werden in Oberrandläsionen, partielle und komplette Rupturen unterteilt. Am häufigsten sind sie im Zusammenhang mit ausgedehnten Supraspinatussehnenrupturen und bei Läsionen des Rotatorenintervalls. Läsionen der Subskapularissehne werden am besten auf transversalen und anguliert-sagittalen Schnittbildern erkannt.
}
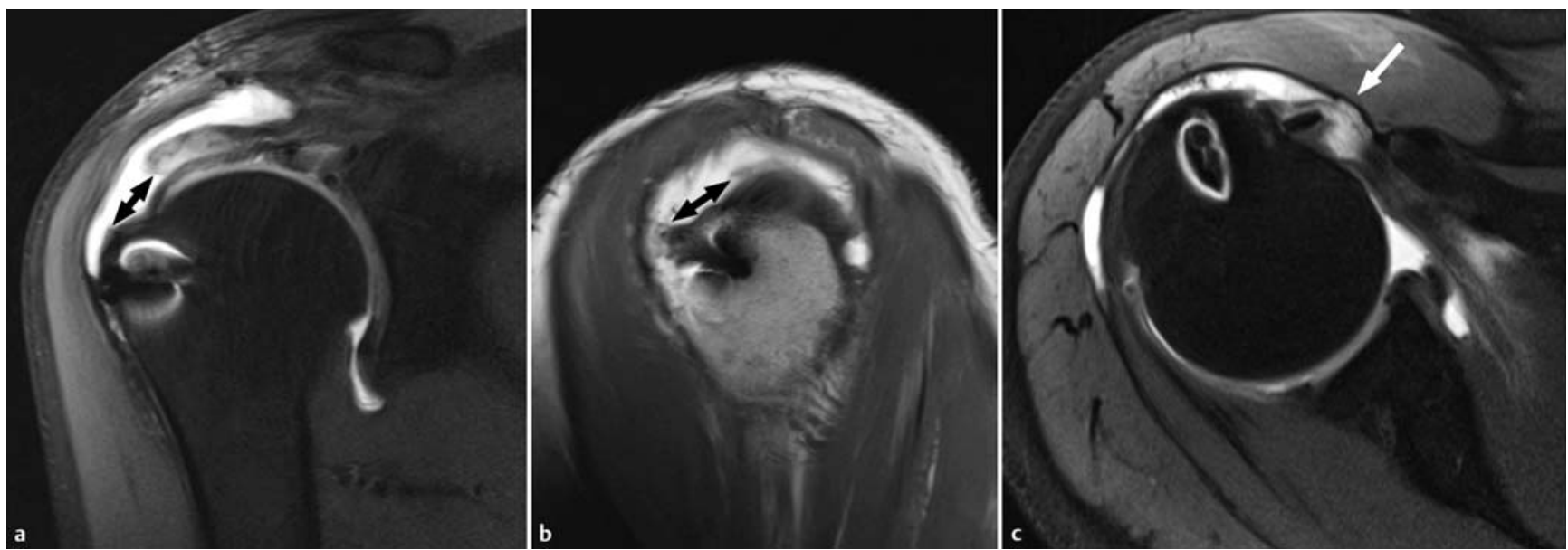

Abb. 14 Reruptur des ventralen Anteils der Supraspinatussehne, direkte MR-Arthrografie. a Die anguliert-koronare, T1w fettunterdrückte Aufnahme zeigt die Größe der Ruptur in mediolateraler Ausdehnung (Doppelpfeil). Es liegt ein Retraktionsgrad 1 vor. b Die anguliert-sagittale, T1w Aufnahme dokumentiert die Ausdehnung der Ruptur in anteroposteriorer Richtung (Doppelpfeil). Aufgrund des Suszeptibilitätsartefaktes ist die Lage der Bizepssehne in dieser Ebene nicht genau beurteilbar. c Die transversale, T1w fettsupprimierte Aufnahme zeigt die Einbeziehung der kranialen Fasern der Subskapularissehne und eine beginnende Medialverlagerung der langen Bizepssehne (Pfeil). 


\section{Postoperative Bildgebung}

OP-Indikationen. Eine chirurgische Behandlung einer Läsion der Rotatorenmanschette ist von vielen Faktoren abhängig, z.B. vom/von:

- Ausmaß der klinischen Beschwerden

- Alter, Aktivitätsniveau des Patienten

- Größe und Ausdehnung der Sehnenläsion

- Ausmaß von Muskelatrophie und -verfettung

- Vorhandensein und Ausmaß von Knorpelveränderungen im Glenohumeralgelenk

So wird eine partielle Rotatorenmanschettenruptur beim alten Patienten eher konservativ, bei einem jüngeren Patienten mit Einschränkung des Aktivitätsniveaus eher operativ versorgt. Generell ist dabei ein Trend zur arthroskopisch gestützten Operation zu verzeichnen [26].

MR-Verfahren. Erneute Rupturen treten zumeist zwischen 6 und 26 Wochen nach einer operativen Versorgung auf und gehen i.d.R. mit einer klinischen Verschlechterung einher [2]. Zur Bildgebung der operierten Rotatorenmanschette können sowohl eine native Untersuchung wie auch eine MR-Arthrografie durchgeführt werden (Abb. 14). Letztere erlaubt zweifelsohne eine bessere Beurteilung kompletter Sehnenrupturen. Auf der anderen Seite ist bekannt, dass kleineren Rupturen $(<1 \mathrm{~cm})$ u.U. keine relevante Bedeutung für das klinische Outcome des Patienten zukommt [26]. Zusätzlich zeigt die MR-Arthrografie Limitationen im Nachweis postoperativer partieller Rupturen, sodass primär eine native Untersuchung zur Beurteilung der postoperativen Rotatorenmanschette als ausreichend angesehen wird. Sie gestattet eine Beurteilung der Integrität der operierten Sehnen, welche nach einer umfänglichen Metaanalyse eine hohe Inter- und Intraobserver-Übereinstimmung aufweist [27]. Sind etwaige Knochenanker zur Refixierung ausgerissener Sehnen vorhanden, sollten Sequenzen verwendet werden, die Metallartefakte reduzieren [28]. Eine Unterdrückung des Fettsignals wird dann besser mit Inversionspulsen als mit einer frequenzselektiven Unterdrückung erreicht.
Befunde. Postoperativ kann innerhalb der ersten 3 Monate eine diffuse Signalanhebung der Sehnenplatte vorhanden sein. Die Diagnose einer erneuten, kompletten Ruptur stützt sich postoperativ auf den Nachweis eines flüssigkeitsäquivalenten Signals, das die gesamte Sehnendicke durchsetzt. Wichtig für die weitere Therapieplanung ist in diesem Fall die Beurteilung der betroffenen Muskeln im Hinblick auf Atrophie und Verfettung, da bei einem Verfettungsgrad $>2$ nach Goutallier ein Ausschlusskriterium für eine anatomische Rekonstruktion der Rotatorenmanschette vorliegt.

Für die postoperative Beurteilung der Rotatorenmanschette ist zumeist eine native MRT ausreichend. Sie erlaubt eine zuverlässige Aussage darüber, ob eine relevante komplette Reruptur vorliegt. Je nach angewendeter Operationstechnik sollten u.U. Techniken zum Einsatz kommen, die Metallartefakte reduzieren.

\section{Audio-Podcast des kompletten Artikels}

Diesen Beitrag können Sie in der HTML-Datei auch als Podcast anhören. Online finden Sie den Beitrag unter www.thieme-connect.de/products.

\begin{abstract}
The rotator cuff consists of the tendons of the supscapularis, supraspinatus, infraspinatus and teres minor muscles. This group of muscles performs multiple functions and is often stressed during various activities. This explains, why rotator cuff disease is common and the most often cause of shoulder pain and dysfunction in adults. MR imaging still is the most important imaging modality in assessment of rotator cuff disease. It enables the radiologist to make an accurate diagnosis, the basis for an appropriate management. In this article, current concepts with regard to anatomy and imaging diagnosis will be reviewed. The discussion of the complex anatomy is followed by normal and pathologic MR imaging appearances of the rotator cuff including tendinopathy and tearing, and concluding with a review of the postoperative cuff.
\end{abstract}

\section{Keywords}

Shoulder · rotator cuff · anatomy · pathology ·

MR imaging 


\section{Kernaussagen}

- Die Rotatorenmanschette der Schulter wird von anterior nach posterior gebildet aus den Sehnen der Mm. subscapularis, supraspinatus, infraspinatus und teres minor. Die Lücke zwischen dem Oberrand der Subskapularis- und dem Vorderrand der Supraspinatussehne wird als Rotatorenintervall bezeichnet. Anatomische Leitstruktur ist die lange Bizepssehne mit ihrem Halteapparat („Pulley“).

- Die Sehnen der Rotatoren weisen einen hochorganisierten Aufbau bestehend aus Bindegewebsfaszikeln auf, deren gegenseitige Verschieblichkeit durch Lubrizin gewährleistet wird. Das zumeist abgrenzbare „rotator cable“ macht aus der Rotatorenmanschette im Bereich der Supra- und Infraspinatussehne eine Hängebrückenkonstruktion und hilft, Zug- und Scherbelastungen zu minimieren.

- Die Rotatorenmanschette kann MRtomografisch sowohl nativ als auch mittels direkter oder indirekter MR-Arthrografie beurteilt werden. Kontrastver- stärkte Untersuchungstechniken sind der nativen Untersuchung insbesondere im Nachweis partieller Rupturen überlegen.

- Tendinopathien und Tendinitiden führen zu einer erhöhten Signalintensität der betroffenen Sehnen. Dies muss gegenüber einem „Magic-Angle“-Artefakt abgegrenzt werden, der sich durch Wahl von Sequenzen mit einer Echozeit $\geq 37$ ms verhindern lässt. Bei einer Tendinitis können nahezu flüssigkeitsäquivalente Signalanhebungen vorgefunden werden, ohne dass eine Unterbrechung der Sehnenkontinuität vorliegt.

- Partielle Rotatorenmanschettenrupturen betreffen nicht den gesamten Querschnitt der Sehne. Man unterscheidet gelenk- von bursaseitig gelegenen sowie interstitielle Partialrupturen. Partialrupturen, die den Ansatz der Sehne mit einbeziehen, werden als „Footprint“-Läsionen bezeichnet.

- Bei der kompletten Rotatorenmanschettenruptur ist der gesamte Sehnenquerschnitt von der Ruptur betroffen.
Rotatorenmanschettenrupturen können zu Atrophie und Verfettung der betroffenen Muskeln führen. Zur Beurteilung der Verfettung hat sich die Einteilung nach Goutallier, zur Beurteilung der Atrophie das Tangentenzeichen nach Zanetti bewährt.

- Die Läsionen der Sehne des M. subscapularis werden in Oberrandläsionen, partielle und komplette Rupturen unterteilt. Sie werden am häufigsten im Zusammenhang mit ausgedehnten Supraspinatussehnenrupturen und bei Läsionen des Rotatorenintervalls vorgefunden.

- Für die postoperative Beurteilung der Rotatorenmanschette ist zumeist eine native MRT ausreichend. Sie erlaubt eine zuverlässige Aussage darüber, ob eine relevante komplette Reruptur vorliegt. Je nach angewendeter Operationstechnik sollten u. U. Techniken zum Einsatz kommen, die Metallartefakte reduzieren.

\section{Über die Autoren}

\section{Karl-Friedrich Kreitner}

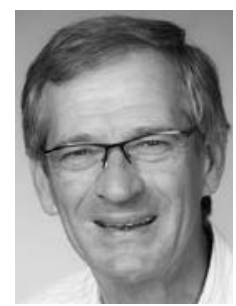

Prof. Dr. med. 1978-1985 Studium der Humanmedizin an der JohannesGutenberg-Universität Mainz. 1986 Promotion. 1985- 1991 Assistenzarzt an der Klinik und Poliklinik für Radiologie der Universitätsklinik Mainz, seit 1993 Oberarzt. 2002 Habilitation. 2008 apl. Professor der JohannesGutenberg-Universität Mainz.

\section{Aline Mähringer-Kunz}

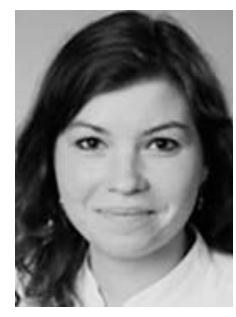

Studium der Humanmedizin an der Philipps-Universität Marburg. Seit 2012 Assistenzärztin an der Klinik und Poliklinik für diagnostische und interventionelle Radiologie des Universitätsklinikums Mainz. Klinische Schwerpunkte im Bereich der muskuloskelettalen Radiologie.

\section{Korrespondenzadresse}

Prof. Dr. Karl-Friedrich Kreitner

Universitätsmedizin Mainz

Klinik und Poliklinik für Diagnostische und

Interventionelle Radiologie

Langenbeckstraße 1

55131 Mainz

Tel. +496131174160

Fax: +496131176633

E-Mail: karl-friedrich.kreitner@unimedizin-mainz.de

Interessenkonflikt: kein Interessenkonflikt angegeben 


\section{Literatur}

1 Yablon CM, Jacobson JA. Rotator cuff and subacromial pathology. Semin Musculoskelet Radiol 2015; 19: 231 - 242

2 Chang EY, Chung BC. Current concepts on imaging diagnosis of rotator cuff disease. Semin Musculoskelet Radiol 2014; 18: $412-424$

3 Opsha O, Malik A, Baltazar R et al. MRI of the rotator cuff and internal derangement. Eur J Radiol 2008; 68: 36 - 56

4 Mochizuki T, Sugaya H, Uomizu M et al. Humeral insertion of the supraspinatus and infraspinatus. New anatomical findings regarding the footprint of the rotator cuff. J Bone Joint Surg Am 2008; 90: $962-969$

5 Llopis E, Montesinos P, Guedez MT et al. Normal shoulder MRI and MR arthrograpjy: anatomy and technique. Semin Musculoskelet Radiol 2015; 19: 212-230

6 Woertler K. Rotator interval. Semin Musculoskelet Radiol 2015; 19: $243-253$

7 Funakoshi T, Schmid T, Hsu H-P et al. Lubricin distribution in the goat infraspinatus tendon: a basis for interfascicular lubrication. J Bone Joint Surg Am 2008; 90: 803 - 814

8 Kassarjian A, Torriani M, Ouellette $\mathrm{H}$ et al. Intramuscular rotator cuff cysts: association with tendon tears on MRI and arthroscopy. Am J Roentgenol 2005; 185: 160-165

9 Gyftopoulos S, Bencardino J, Nevsky G et al. Rotator cable: MRI study of its appearance in the intact rotator cuff with anatomic and histologic correlation. Amer J Roentgenol 2013; 200: $1101-1105$

10 Burkhart SS, Esch JC, Jolson RS. The rotator crescent and rotator cable: an anatomic description of the shoulder's "suspension bridge". Arthroscopy 1993; 9: 611-616

11 Mesiha MM, Derwin KA, Sibole SC et al. The biomechanical relevance of anterior rotator cuff cable tears in a cadaveric shoulder model. J Boint Joint Surg Am 2013; 95: 1817 - 1824

$12 \mathrm{Choo} \mathrm{HJ}$, Lee SJ, Kim J-H et al. Delaminated tears of the rotator cuff: prevalence, characteristics, and diagnosic accuracy using indirect MR arthrography. Am J Roentgenol 2015; 204: $360-366$

13 De Jesus JO, Parker L, Frangos AJ et al. Accuracy of MRI, MR arthrography, and ultrasound in the diagnosis of rotator cuff tears: a meta-analysis. Am J Roentgenol 2009; 192: 1701 1707

14 Lee JH, Yoon YC, Jee S. Diagnostic performance of indirect MRarthrography for the diagnosis of rotator cuff tears at 3.0 T. Acta Radiol 2015; 56: 720-726
15 Schreinemachers SA, van der Hulst VPM, Willems WJ et al. Detection of partial-thickness supraspinatus tendon tears: is a single direct MR arthrography series in ABER position as accurate as conventional MR arthrography? Skeletal Radiol 2009; 38: $967-975$

16 Zanetti M, Mamisch-Saupe N. Magnetresonanztomographie der Schulter. Impingement und instabilitätsassoziierte Veränderungen - Update 2013. Radiologe 2013; 53: 917 - 933

17 Schaeffeler C, Mueller D, Kirchhoff C et al. Tears at the rotator cuff footprint: prevalence and imaging characteristics in 305 arthrograms of the shoulder. Eur Radiol 2011; 21: 1477 1484

18 Ellman H. Diagnosis and treatment of incomplete rotator cuff tears. Clin Orthop Relat Res 1990; 254: 64 - 74

19 Waldt S, Eiber M, Wörtler K. Messverfahren und Klassifikationen in der muskuloskelettalen Radiologie. Stuttgart: Thieme; 2011

20 Patte D. Classification of rotator cuff lesions. Clin Orthop Relat 1990; 254: $81-86$

21 Goutallier D, Postel JM, Bernageau J et al. Fatty muscle degeneration in cuff ruptures. Pre- and postoperative evaluation by CT scan. Clin Orthop Relat Res 1994; 304: 78 - 83

22 Fuchs B, Weishaupt D, Zanetti M et al. Fatty degeneration of the muscles of the rotator cuff: assessment by computed tomography versus magnetic resonance imaging. J Shoulder Elbow Surg 1999; 8: 599-605

23 Zanetti M, Gerber C, Hodler J et al. Quantitative assessment of the muscles of the rotator cuff with magnetic resonance imaging. Invest Radiol 1998; 33: 163-170

24 Pfirrmann CWA, Zanetti M, Weishaupt D et al. Subscapularis tendon tears: detection and grading at MR arthrography. Radiology 1999; 213: 8709-8714

25 Gyftopoulos S, O'Donnell J, Shah NP et al. Correlation of MRI with arthroscopy for the evaluation of the subscapularis tendon: a musculoskeltal division's experience. Skeletal Radiol 2013; 42: 1269-1275

26 Woertler K. Multimodality imaging of the postoperative shoulder. Eur Radiol 2007; 17: 3038 - 3055

27 Saccomanno MF, Cazzato G, Fodale M et al. Magnetic resonance imaging criteria for the assessment of the rotator cuff after repair: a systematic review. Knee Surg Sports Traumatol Arthrosc 2015; 23: 423-442

28 Beltran LS, Bencardino JT, Steinbach LS. Postoperative MRI of the shoulder. J Magn Reson Imaging 2014; 40: 1280 - 1297 


\section{CME-Fragen}

Die folgenden Fragen beziehen sich auf den vorangehenden Beitrag. Bitte schicken Sie uns die entsprechenden Lösungsbuchstaben. Jeweils eine Antwort ist richtig. Die Vergabe von CME-Punkten ist an die korrekte Beantwortung der Multiple-Choice-Fragen gebunden.

\section{1}

Welche der folgenden Aussagen ist nicht richtig?

A Zur Rotatorenmanschette zählen die Sehnen der Mm. subscapularis, supraspinatus, infraspinatus und teres minor.

B Die Rotatorenmanschette zählt zu den wichtigsten passiven Stabilisatoren des Schultergelenks.

C Erkrankungen der Rotatorenmanschette zählen im Erwachsenenalter zu den häufigsten Ursachen von Schmerzen im Schultergelenk.

D Eine wichtige Funktion der Rotatoren des Schultergelenks ist das Zentrieren des Oberarmkopfes in der Gelenkpfanne bei Bewegungen im Schultergelenk.

E Kennzeichen der Rotatoren des Schultergelenks ist der Ursprung an der Skapula und der Ansatz am Humeruskopf.

2

Welche der Sehnen der Rotatorenmanschette ist von Verletzungen und Veränderungen am häufigsten betroffen?
A Subskapularissehne
B Supraspinatussehne
C Infraspinatussehne
D Teres-minor-Sehne
E Die Sehnen sind gleich häufig betroffen.

\section{3}

Welche der folgenden Aussagen ist richtig?
A Als Rotatorenintervall wird üblicherweise die Lücke zwischen dem Hinterrand der Sehne des M. supraspinatus und dem Vorderrand der Sehne des M. infraspinatus bezeichnet.

B Anatomische Leitstruktur des Rotatorenintervalls ist die Sehne des M. supraspinatus.

C Läsionen des Rotatorenintervalls spielen klinisch keine Rolle.

D Das Rotatorenintervall wird medial von der Basis des Korakoidfortsatzes begrenzt und läuft nach lateral in den Sulcus intertubercularis und das Lig. transversum humeri aus.

E Pulley-Läsionen sind zumeist mit Verletzungen der Supraspinatussehne assoziiert.
4

Welche der folgenden Aussagen ist nicht richtig?
A Die Sehnen der Rotatoren bestehen aus hochorganisierten Kollagenfibrillen, die zu Faszikeln angeordnet sind.

B Lubrizin ermöglicht ein Gleiten der Faszikel gegeneinander.

C Im Bereich der Supra- und Infraspinatussehne können histologisch 5 Schichten differenziert werden.

D Das „rotator cable“ entspricht einem tiefen Ausläufer des Lig. coracohumerale.

E Das „rotator cable“ ist üblicherweise auf anguliert-koronaren MRT-Aufnahmen bei allen Untersuchten abgrenzbar. 


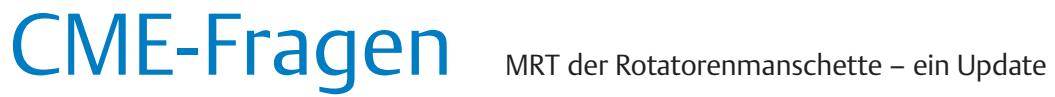

5

Welche der folgenden Aussagen ist richtig?
A Bei der MRT der Rotatorenmanschette kommen üblicherweise gespoilte Gradienten-EchoSequenzen zum Einsatz.

B Das „Magic-Angle“-Phänomen entsteht bei geordneten anatomischen Strukturen wie z. B. Sehnenfasern dann, wenn deren Ausrichtung einen Winkel von etwa $55^{\circ}$ gegenüber dem statischen Magnetfeld einnimmt.

C Das „Magic-Angle“-Phänomen kann vor allem bei der Beurteilung der Subskapularissehne eine Rolle spielen.

D Bei protonendichtegewichteten schnellen Spin-Echo-Sequenzen mit Echozeiten unter 35 ms spielt das „Magic-Angle“-Phänomen keine Rolle.

E Für eine suffiziente Beurteilung der Rotatorenmanschette sind Aufnahmen in anguliert-koronarer und transversaler Schnittführung ausreichend.

\section{6}

Welche der folgenden Aussagen ist nicht richtig?
A Bei einer Tendinopathie können histologisch mukoide Degenerationen und eine Anhäufung von Glykosaminoglykanen vorliegen.

B Tendinopathien weisen eine erhöhte Signalintensität auf Aufnahmen mit kurzen Echozeiten auf.

C Diese müssen von dem „Magic-Angle“-Phänomen abgegrenzt werden.

D Entzündungen der Sehnen können durch den erhöhten Wassergehalt auch auf T2w Aufnahmen eine deutlich erhöhte Signalintensität aufweisen.

E Die native MRT ist der direkten MR-Arthrografie in der Differenzierung zwischen ausgedehnten Tendinitiden, Partialrupturen und kleinen kompletten Sehnenrupturen ebenbürtig.
7

Welche der folgenden Aussagen ist nicht richtig?
A Partielle Rotatorenmanschettenrupturen betreffen definitionsgemäß den ganzen Sehnenquerschnitt, aber nicht die gesamte Sehne.

B Man unterscheidet gelenk- von bursaseitig gelegenen sowie interstitielle Partialrupturen.

C Partialrupturen, die den Ansatz der Sehne mit einbeziehen, werden als „Footprint“-Läsionen bezeichnet.

D „Footprint“-Läsionen finden sich eher bei jüngeren Patienten und werden eher einer operativen Therapie zugeführt.

E Die direkte MR-Arthrografie verbessert signifikant die Sensitivität der MRT im Nachweis partieller Rotatorenmanschettenrupturen.

\section{8}

Welche der folgenden Aussagen ist richtig?
A Bei einer kompletten Rotatorenmanschettenruptur spielen für die weitere Therapie die Größe der Ruptur und das Ausmaß der Sehnenretraktion keine Rolle.

B Bei einer Rotatorenmanschettenruptur, bei der die gesamte Sehne abgerissen ist, spielt der Zeitpunkt einer etwaigen operativen Versorgung keine Rolle, da Verfettungen und eine Atrophie des betroffenen Muskels erst nach mehreren Monaten auftreten.

C Das Tangentenzeichen ist hilfreich für den Nachweis einer Atrophie des M. supraspinatus.

D Für die Beurteilung einer Verfettung der Rotatoren sollten Aufnahmen in anguliert-koronarer Schnittführung herangezogen werden.

E Die Subskapularissehne ist am häufigsten von einer kompletten Ruptur betroffen. 


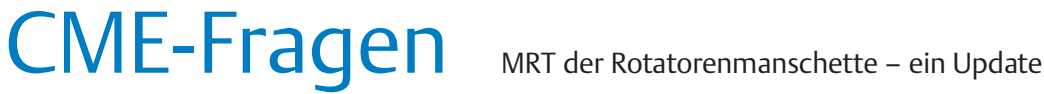

9

Welche der folgenden Aussagen ist nicht richtig?
A Die Sehne des M. subscapularis wird am besten auf transversalen und anguliert-sagittalen Aufnahmen beurteilt.

B Als Oberrandläsion wird eine Läsion bewertet, bei der das kraniale Drittel der Sehne betroffen ist.

C Traumatische Rupturen der Subskapularissehne sind zumeist Folge eines Außenrotationstraumas bei adduziertem Arm.

D Bei pathologischen Veränderungen des Oberrandes der Subskapularissehne sollte auch auf Verletzungen des Rotatorenintervalls und der langen Bizepssehne geachtet werden.

E Die fettige Atrophie des M. subscapularis ist ein sensitives Zeichen für das Vorliegen einer Subskapularissehnenruptur.

10

Welche der folgenden Aussagen ist richtig?
A Postoperativ sollte bei Verdacht auf eine Reruptur der Rotatorenmanschette immer eine direkte MR-Arthrografie erfolgen.

B Postoperativ werden bevorzugt Gradienten-Echo-Sequenzen angewendet.

C Je nach angewendeter Operationstechnik sollten ggf. Sequenztechniken zum Einsatz kommen, die Metallartefakte reduzieren.

D Postoperativ spielt der Verfettungsgrad der Rotatoren keine Rolle bei der Planung einer etwaigen erneuten Operation.

E Aufnahmen in Abduktion und Außenrotation sind äußerst hilfreich im Nachweis von Rerupturen der Rotatorenmanschette.

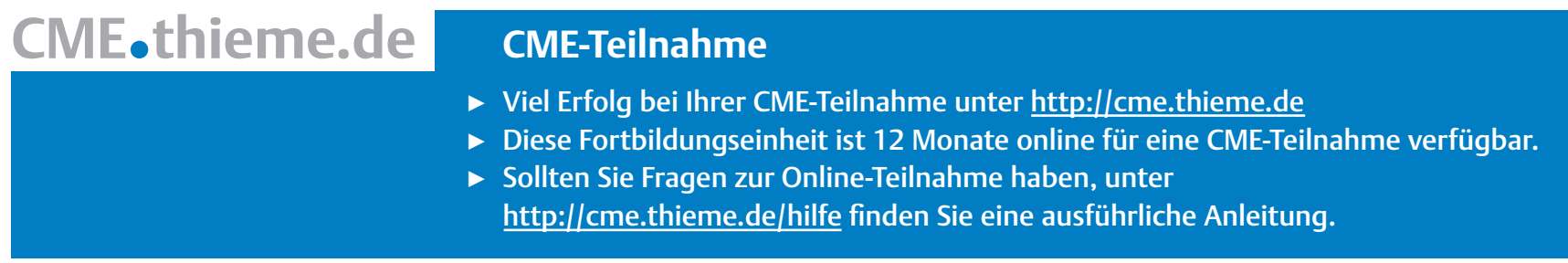

